\title{
Exenatide reverts the high-fat-diet-induced impairment of BDNF signaling and inflammatory response in
} an animal model of Alzheimer's disease

Manuela Bomba ${ }^{1,2, *}$, Alberto Granzotto ${ }^{1,2, *}$, Vanessa Castelli ${ }^{3}$, Rossano Lattanzio ${ }^{1,4}$, Annamaria Cimini ${ }^{3,5,6}$, Stefano L. Sensi ${ }^{1,2,7, \#}$

${ }^{1}$ Center of Excellence on Aging and Translational Medicine - CeSI-MeT, University G. d'Annunzio of ChietiPescara, Italy

${ }^{2}$ Department of Neuroscience, Imaging, and Clinical Sciences, University G. d'Annunzio of Chieti-Pescara, Italy

${ }^{3}$ Department of Life, Health and Environmental Sciences, University of L'Aquila, Italy

${ }^{4}$ Department of Medical, Oral, and Biotechnological Sciences, University G. d'Annunzio of Chieti-Pescara, Italy

${ }^{5}$ Sbarro Institute for Cancer Research and Molecular Medicine and Center for Biotechnology, Temple University, Philadelphia, USA

${ }^{6}$ National Institute for Nuclear Physics (INFN), Gran Sasso National Laboratory (LNGS), Assergi, Italy

${ }^{7}$ Departments of Neurology and Pharmacology, Institute for Mind Impairments and Neurological Disorders

- iMIND, University of California - Irvine, Irvine, USA

* Co-first author

\# Corresponding author:

Prof. Stefano L. Sensi

Center of Excellence on Aging and Translational Medicine - CeSI-MeT,

University G. d'Annunzio of Chieti-Pescara,

Via Colle dell'Ara, Chieti 66100, Italy.

Tel.: +390871 541544;

fax: +390871 541542;

e-mail: ssensi@uci.edu 


\section{Abstract}

Background: preclinical, clinical, and epidemiological evidence support the notion that Alzheimer's disease $(A D)$ is a multifactorial condition in which, along with $\beta$-amyloid $(A \beta)$ and tau-related pathology, the synergistic activity of genetic, environmental, vascular, metabolic, and inflammatory factors promote the onset and progression of the disease. Epidemiological evidence indicate that glucose intolerance, deficits in insulin secretion or type 2 diabetes mellitus (T2DM) participate in increasing the risk of developing cognitive impairment or dementia. A pivotal role in the process is played by insulin as the hormone critically regulates brain functioning. GLP-1, the glucagon-like peptide 1, facilitates insulin signaling, regulates glucose homeostasis, and modulates synaptic plasticity. Exenatide is a GLP-1R agonist, characterized by an extended half-life, employed in T2DM. However, exenatide has also been shown to affect the signaling of the brain-derived neurotrophic factor (BDNF), synaptic plasticity, and cognitive performances in animal models of brain aging and neurodegeneration.

Methods: in this study, we tested whether exenatide exerts neuroprotection in a preclinical AD model set to mimic the clinical complexity of the human disease. To that aim, we investigated the effects of 3-month exenatide treatment in 3xTg-AD mice challenged for six months with a high-fat diet (HFD). Endpoints of the study were variations in systemic metabolism, insulin and neurotrophic signaling, neuroinflammation, levels of $A \beta$ and tau pathology as well as changes in cognitive performances.

Findings and interpretation: results of the study indicate that exenatide reverts the adverse changes of BDNF signaling and the neuroinflammation status of 3xTg-AD mice undergoing HFD.

Keywords: exendin-4, insulin, diabetes, T2DM, insulin resistance, BDNF, neurotrophic factors, dementia, aging, synaptic plasticity, memory. 


\section{Introduction}

Alzheimer's disease (AD) is a neurodegenerative condition associated with the presence of cognitive and behavioral deficits and characterized by the accumulation of neuritic plaques composed of $\beta$-amyloid (A $\beta$ ) peptides, the appearance of neurofibrillary tangles (NFT) made of hyperphosphorylated tau ( $p$-tau) proteins, and reactive gliosis ${ }^{1}$. Preclinical, clinical, and epidemiological data support the notion that $A D$ is a multifactorial condition in which, along with $A \beta$ and tau-related pathology, the convergence of genetic, environmental, vascular, metabolic, and inflammatory factors promotes the onset and development of the disease ${ }^{2-7}$. In that regard, metabolic disorders are the objects of growing therapeutic attention ${ }^{8-11}$. Epidemiological studies indicate that glucose intolerance, deficits in insulin secretion or type 2 diabetes (T2DM) increase the risk of developing cognitive impairment or dementia ${ }^{11}$.

In the Central Nervous System (CNS), insulin regulates many critical biological events occurring in neurons, glia, microglia, and the neurovascular unit. In that compartments, insulin participates in the control of energy homeostasis, the modulation of protein and lipid synthesis, apoptosis, autophagy, the permeability of the blood-brain barrier, neurotransmitter balance, cytoskeletal remodeling, synaptic plasticity and neurogenesis ${ }^{11-13}$. Insulin resistance (IR), a condition observed in T2DM patients, has also been described in the brain of AD patients. In the brain, IR can occur even in the absence of obesity, peripheral IR or overt signs of T2DM ${ }^{14}$. In the CNS, IR leads to impaired structural and functional plasticity, ultimately contributing to the development of neuronal and brain dysfunctions ${ }^{11,15,16}$.

GLP-1, the glucagon-like peptide 1, is a hormone that facilitates insulin signaling and regulates glucose homeostasis ${ }^{17,18}$. However, GLP-1 receptors (GLP-1Rs) are also expressed in the brain ${ }^{17}$ where they contribute to the modulation of neuronal excitability, synaptic plasticity, and cognition ${ }^{19-21}$. Exenatide is a GLP-1R agonist, characterized by an extended half-life, and employed in T2DM. The molecule also promotes beneficial effects in the CNS $3,22,23$. In preclinical models of brain aging and neurodegeneration, exenatide has been shown to positively affect the signaling of the brain-derived neurotrophic factor (BDNF) and to modulate synaptic plasticity and cognitive performances ${ }^{24-27}$. In clinical settings, exenatide has been successfully employed to alleviate the motor symptoms of Parkinson's disease (PD) patients ${ }^{28,29}$.

In this study, we tested whether exenatide promotes neuroprotection in a preclinical AD model that was set to mimic the clinical complexity of the human disease. To that aim, we evaluated the effects of a 3-month treatment in 3XTg-AD mice that were challenged for six months with a high-fat diet (HFD). Endpoints of the study were variations in systemic metabolism, insulin, and neurotrophic signaling, neuroinflammation, changes in levels of $A \beta$ - and tau-pathology or cognitive performances. Age-matched 3XTg-AD animals fed with a standard diet or undergoing HFD and treated with vehicle were used as controls. 


\section{Materials and Methods}

\section{Animals and treatment paradigm}

All the procedures involving the animals and their care were approved by the Local Institutional Ethics Committee (Comitato Etico Interistituzionale per la Sperimentazione Animale [CEISA] protocol no. 17; Min. IDD: DGSAF/14264). Animal handling was performed in compliance with national and international laws and policies. All efforts were employed to reduce the number of animals and their suffering upon all the experimental procedures. 3xTg-AD mice [B6;129-Tg(APPSwe,tauP301L)1Lfa Psen $^{\text {tm } 1 \mathrm{Mpm}} / \mathrm{Mmjax}$ ] were purchased from Jackson Laboratory, bred in the Center of Excellence on Aging and Translational Medicine (CeSI-MeT) animal facility, housed on 12-12 hours light/dark cycle, and provided with, until treatment allocation, ad libitum access to standard chow and water. A total of forty-six 3xTg-AD mice (21 males and 25 females) were enrolled at six months of age (m.o.a.) and randomly assigned to a 6month control or high fat dietary regimen. 3-month after the beginning of the dietary treatment, mice of the control $\left(3 x T g-A D^{C D}\right)$ and high fat $\left(3 x T g-A D^{H F D}\right)$ groups were randomly assigned to a 3-month administration of exenatide or vehicle (PBS). HFD was purchased from Altromin. In this obesity-inducing chow, $60 \%$ of the energy derives from fats. CD consists of a standard chow with $13 \%$ of energy deriving from fats. Exenatide or vehicle administration were performed as previously described ${ }^{26}$. Briefly, exenatide ( $500 \mu \mathrm{g} / \mathrm{kg}$ body weight) or vehicle were administered via intraperitoneal injection five days per week. Treatments were also maintained during the behavioral testing phase. The exenatide lyophilized powder was provided by Eli Lilly.

\section{Insulin sensitivity and glucose tolerance tests}

Insulin sensitivity and glucose tolerance were assessed at 6, 9, and 12 m.o.a. by employing the intraperitoneal insulin tolerance test (ITT) and the glucose tolerance test (GTT), respectively. For ITT, after a six-hour fasting period, mice were injected with 0.75 unit/kg of human insulin (Sigma-Aldrich). For, GTT, after an overnight fasting period $(\approx 16 \mathrm{~h}$ ), mice were injected with $1 \mathrm{~g} / \mathrm{kg}$ glucose (Sigma-Aldrich). Glycemia was measured from vein tail blood drop with a Freestyle InsuLinx glucometer (Abbott). Measurements were performed 5, 15 30, 45, 60, 120, and 180 min after the insulin or glucose administration. Insulinrelated measurements were halted at the $30^{\text {th }}$ min to avoid hypoglycemia.

\section{Plasma insulin assay and HOMA-IR assessment}

Plasma insulin concentrations were determined with the Ultrasensitive Insulin ELISA kit (Mercodia) following the manufacturer instructions. Fasting glucose and insulin concentrations for the Homeostasis Model Assessment of Insulin Resistance (HOMA-IR) calculations were employed as [insulin ( $\mathrm{pM} / \mathrm{L}$ ) X glucose $(\mathrm{mM} / \mathrm{L}) / 22.5]$. 


\section{Tissue collection}

At the end of treatments and following behavioral tests and metabolic analyses, mice were anesthetized, killed, and tissue samples harvested for biochemical analysis. Brains were halved into 2 hemispheres. For immunohistochemical (IHC) analyses, one hemisphere was collected in a Carnoy solution, kept for 2 days at $4^{\circ} \mathrm{C}$, ethanol-washed, and paraffin-embedded until sectioning. For Western blot (WB) analysis, each hemisphere was dissected into subregions (hippocampus, whole cortex, and cerebellum), snap-frozen in liquid nitrogen, and stored at $-80^{\circ} \mathrm{C}$ until sampling.

\section{$A B$ and $p$-tau immunohistochemistry}

Five $\mu \mathrm{m}$ sections of Carnoy-fixed and paraffin-embedded brains of 3xTg-AD mice from the four experimental groups were stained using purified mouse monoclonal antibodies raised against human $A \beta$ (clone DE2B4, 1:200 dilution, overnight incubation, Abcam) and $\mathrm{p}$-tau ( $\mathrm{Thr}_{231}$; AT180, Pierce Protein Research Products) as previously reported ${ }^{25}$. Briefly, antigen retrieval was performed in $10 \mathrm{mmol} / \mathrm{l}$ sodium citrate buffer ( $\mathrm{pH} \mathrm{6.0)}$ by a thermostatic bath at $100^{\circ} \mathrm{C}$ for $10 \mathrm{~min}$ for $A \beta$ and by microwave treatment at $750 \mathrm{~W}$ for $10 \mathrm{~min}$ for $\mathrm{p}$-tau. The anti-mouse EnVision kit (Agilent) was used for signal amplification. In control sections, the specific primary antibodies were replaced with isotype-matched immunoglobulins. In the hippocampus, $A \beta$ deposition was quantified by counting the number of $A \beta$ plaques using a $10 x$ magnification. The immunostaining signal evaluation for $p$-tau was performed by counting stained pixels on hippocampal neurons using Photoshop (Adobe Systems) as previously reported ${ }^{25}$.

\section{Western blot analysis}

Brain regions were lysed in ice-cold RIPA buffer containing [in \%]: 0.5 sodium deoxycholate, 1 Nonidet P-40, 0.1 SDS, 1 protease and phosphatase inhibitor cocktails, and $5 \mathrm{mM}$ EDTA, pH 7.4. Protein lysates $(10 \mu \mathrm{g})$ were separated on a SDS-polyacrylamide gel (9\%-13\% gradient) and blotted onto PVDF membrane. Nonspecific binding sites were blocked with 5\% non-fat dry milk (Bio-Rad Laboratories) in Trisbuffered saline (TBS) containing [in mM]: 20 Tris- $\mathrm{HCl}, 150 \mathrm{NaCl}, \mathrm{pH}$ 7.4, for 30 minutes at room temperature. Membranes were incubated overnight at $4{ }^{\circ} \mathrm{C}$ with the primary antibodies, diluted with TBS supplemented with $0.1 \%$ Tween 20 (TBS-T) and $5 \%$ nonfat dry milk. A list of the WB employed antibodies and their dilution is available in the Supplementary Table S1. Peroxidase-conjugated secondary anti-rabbit or anti-mouse IgG antibodies (1:10,000; Vector Laboratories) were used. Chemiluminescent signals were visualized by enhanced chemiluminescence (EuroClone), following the manufacturer instructions. Relative densities were determined and normalized to a housekeeping protein (actin) using ImageJ software. Values are given as relative units or phosphoprotein/total protein ratio, and calculated as: (phosphoprotein/loading control)/(total protein/loading control). 


\section{Morris water maze test}

The Morris Water Maze (MWM) test was performed as previously described ${ }^{30}$. Briefly, the MWM apparatus (Panlab) consists in a circular pool (1.2 $\mathrm{m}$ diameter) filled with warm water $\left(22 \pm 1^{\circ} \mathrm{C}\right)$. The pool is placed in a noise-isolated room containing several intra- and extra-maze visual cues. Mice were trained to swim in the pool and reach a circular platform located $2 \mathrm{~cm}$ beneath the water surface. Mice that failed to find the platform within 90 seconds were manually guided to it and allowed to remain there for 10 seconds. Mice performed four trials per day for four consecutive days. Spatial memory performances were assessed 1.5 and 24 hours after the end of the last training trial. The probe test consisted of a 60 -second free swim in the pool in which the platform has been removed. Probe tests were recorded for subsequent analysis with the Smart tracking software (Panlab). Performances were evaluated in terms of time employed to reach the location where the platform used to be (escape latency), number of crosses over the platform location, and time spent in the target (T target) quadrant.

\section{The novel object recognition test}

The Novel Object Recognition (NOR) test was performed as previously described ${ }^{31}$. Briefly, in the habituation phase, 3xTg-AD mice were placed for $10 \mathrm{~min}$ per day for 2 consecutive days in an empty cage. On day 3, mice were placed in a cage containing two identical objects spaced $\sim 15 \mathrm{~cm}$ apart and allowed to explore the objects for $8 \mathrm{~min}$. At the end of each trial, objects were thoroughly cleaned with ethanol and air dried. On day 4, mice were placed in the experimental cage containing the objects that have been previously presented. One of these objects was left in the previous location (familiar location) while the other one was placed in a new position (novel location). Mice were then allowed to explore the two objects for $5 \mathrm{~min}$ (probe test). The test was videotaped for subsequent analysis that was manually performed in blind conditions. Scoring of the NOR performance was analyzed in terms of time spent to explore both objects, presented in a familiar location and novel one. Mice were considered explorative when the head was within $1 \mathrm{~cm}$ from the object with the neck extended and vibrissae moving. Proximity or chewing did not count as exploration. Analyzed parameters were the percentage of time spent with the object in the novel location and the discrimination index (DI). DI was calculated as follows: $((A-B) /(A+B)) \times$ 100 , where $A$ is the time spent to explore the object in the novel location and $B$ is the time spent on the object in the familiar one ${ }^{31}$.

\section{Data analysis}

No statistical methods were used to predetermine sample size. Statistical analysis was performed by one-way ANOVA followed by Tukey's post-hoc test. For IHC data Kruskal-Wallis test followed by Tamhane post-hoc test was performed. SPSS Version 15.0 was used throughout. By conventional criteria, 
the level of significance was set at $p<0.05$. Data were expressed as mean \pm standard error of the mean unless otherwise indicated.

\section{Results}

Exenatide treatment has no effect on body weight and glucose metabolism in $3 \times T g-A D^{C D}$ and $3 \times T g-A D^{H F D}$ mice

Male and female $3 \times \operatorname{Tg}-A D$ mice $(n=46)$ at 6 m.o.a. were randomly assigned to a control $\left(3 \times T g-A D^{C D}\right.$; $\mathrm{n}=24$ ) or HFD (3xTg-AD HFD; $n=22$ ) for 6 months. At 9 m.o.a., 3xTg-AD ${ }^{\mathrm{CD}}$ and 3xTg-AD ${ }^{\mathrm{HFD}}$ mice were subjected to intraperitoneal injections of exenatide ( $500 \mu \mathrm{g} / \mathrm{kg}$, five days per week; $\mathrm{n}=11$ and 9 , respectively) or vehicle (saline; $n=13$ for both groups). Compared to $3 x T g-A D^{C D}$ animals, $3 \times T g-A D^{H F D}$ mice exhibited a significant increase in body weight (Fig. 1b; p<0.001). Unexpectedly, no differences in body weight were found when comparing vehicle- and exenatide-treated animals (Fig. 1b; $>0.05$ ).

HFD promotes IR and T2DM ${ }^{32}$. To evaluate the HFD-dependent metabolic changes, glucose metabolism and insulin sensitivity were measured in vehicle- and exenatide-treated $3 \times \operatorname{Tg}-A D^{C D}$ and $3 \times T g-$ $A D^{H F D}$ mice. Mice were sampled at 12 m.o.a. No metabolic differences were observed at 6 or 9 m.o. among the study groups (Supplementary fig. 1). Analysis of the intra-peritoneal GTT, a measure of the body ability to metabolize glucose, showed no diet- or drug-related effects in the study groups (Fig. 1c-d; $\mathrm{p}>0.05$ ). Similarly, no differences were observed in fasting blood glucose levels (Fig. $1 \mathrm{~h} ; \mathrm{p}>0.05$ ). Evaluation of the ITT, to assess the body response to the exogenous administration of insulin, showed that $3 x T g-A D^{H F D}$ mice exhibit altered ITT when compared to $3 x T g-A D^{C D}$ animals (Fig. 1 e-f; $p<0.01$ ). In addition, compared to $3 \times T g-A D^{C D}$ animals, $3 \times T g-A D^{H F D}$ mice showed a mild increase in fasting plasma insulin levels (Fig. 1i; p=0.06). These insulin-related parameters were not affected by the use of exenatide in both diets (Fig. 1e-i; p>0.05), thereby indicating a lack of metabolic effect of the compound.

The HOMA-IR is a mathematical formula that takes into account resting levels of glucose and insulin and provides an indirect estimation of IR. Compared to $3 \times T g-A D^{C D}$ animals, the analysis of this parameter showed a trend toward increased HOMA-IR levels in 3xTg-AD ${ }^{\text {HFD }}$ mice (Fig. 1g; $p=0.06$ ). No exenatidedriven effects were observed in 3xTg-AD $\mathrm{HFD}$ or $3 \times T_{g}-A D^{C D}$ mice (Fig. $1 g ; p<0.05$ ).

\section{$A 6$ - and tau-pathology in $3 \times T g-A D^{C D}$ and $3 \times T g-A D^{H F D}$ mice}

Previous studies have shown that the HFD administration exacerbates the development of $A \beta$ and tau pathology in preclinical AD models including the $3 \times \operatorname{Tg}-A D$ mice ${ }^{33,34}$. The IHC analysis of hippocampal A $\beta$ plaque loads and $p$-tau immunoreactivity of the study animals revealed no differences when comparing 
3XTg-AD ${ }^{C D}$ and $3 \times T$ Tg-AD ${ }^{H F D}$ mice (Fig. 2a-d). In agreement with previous findings ${ }^{25}$, exenatide treatment did not affect $A \beta$ and tau levels in $3 x T g-A D^{C D}$ and $3 x T g-A D^{H F D}$ animals (Fig. 2a-d).

Exenatide treatment positively affects $B D N F$ signaling in $3 x T g-A D^{C D}$ mice and prevents the development of neurotrophic signaling impairment in $3 x T g-A D^{H F D}$ animals

HFD administration impairs BDNF neurotrophic signaling along with the downstream activation of the CAMP response element-binding (CREB) protein, a transcription factor essential for synaptic plasticity and learning and memory processes ${ }^{35,36}$. Compared to $3 \times \operatorname{Tg}-A D^{\mathrm{CD}}$ animals, $W B$ analysis performed on hippocampal, cortical, and cerebellar homogenates obtained from the study animals showed decreased BDNF levels in 3xTg-AD HFD mice (Fig. 3a; $p<0.01$ ). This result was mirrored by an HFD-driven hippocampal reduction of the active forms of key plasticity related modulators. Reduced levels of phosphorylated TrkB ( $p$ TrkB; the high-affinity BDNF receptor), phosphorylated ERK5 (a downstream effector of the BDNF/TrkB axis), and phosphorylated CREB (pCREB; Figs. 3b-d; $p<0.01$ ) were observed in $3 \times T^{2}-A D^{H F D}$ mice. As BDNF signaling is a critical regulator of structural plasticity, we investigated the HFD-related effects on phosphorylated Synapsin I (pSyn) and PSD95, two proteins known to be involved in synapse stabilization at pre- and post-synaptic levels ${ }^{37,38}$. In line with the BDNF results, $3 \times T$ g-AD ${ }^{H F D}$ mice showed a marked decrease of the two proteins when compared to $3 x T g-A D^{C D}$ animals (Fig. 3e-f; $p<0.001$ ).

Compared to vehicle-treated animals, exenatide-treated mice showed a significant increase in BDNF, pERK5, pCREB, pSyn, and PSD95 (Fig. 3a-f). The drug also promoted increased pTrkB levels (Fig. 3c). These changes showed a trend toward statistical significance when analyzed with ANOVA (Tukey post-hoc test; $p=0.09$ ) and were statistically significant when not corrected for multiple comparisons (Student $t$-test; $p=0.02)$. In the $3 x T g-A D^{H F D}$ mice, exenatide prevented the development of HFD-induced impairment of BDNF signaling (Fig. 3a). Exenatide also promoted enhanced BDNF signaling in 3xTg-AD ${ }^{C D}$ mice, thereby suggesting that the molecule produces a neurotrophic drive independently of the dietary regimen (Fig. 3a; $\mathrm{p}<0.001)$.

As GLP-1R signaling modulates insulin-related pathways, we evaluated the insulin receptor substrate 1 (IRS-1) phosphorylation status at serine 1101, a modification known to inhibit downstream insulin signaling ${ }^{39}$. WB analysis of this parameter showed decreased pIRS-1 levels in 3xTg-AD HFD mice $(p<0.001)$, a change that was reverted by exenatide administration (Fig. $3 \mathrm{~g}$ ). No drug-related effects were observed in 3xTg-AD ${ }^{C D}$ animals (Fig. 3g; $>0.05$ ).

Exenatide treatment decreases $p 75$ NTR activation in $3 x T g-A D^{C D}$ mice and prevents the neurotoxic signaling in $3 \times T g-A D^{H F D}$ animals

The biologically mature, and thus active, form of BDNF originates from the proteolytic cleavage of proBDNF. In contrast with the plasticity effects of BDNF, proBDNF, acting on the high-affinity P75 
neurotrophin receptor (P75NTR), activates apoptotic signaling ${ }^{40}$. WB analysis of hippocampal, cortical, and cerebellar lysates of the study groups showed increased proBDNF levels in $3 \times T^{-}$-AD ${ }^{\mathrm{HFD}}$ mice (Fig. 4a; $\mathrm{p}<0.01$ ). HFD promoted p75NTR overexpression and phosphorylation as well as activation of JNK ( $p J N K$ ) and $E_{1,2}\left(p E R K_{1,2}\right)$, two downstream effectors of the proBDNF/p75NTR signaling cascade (Fig. 4b-d; $\mathrm{p}<0.01)$. Compared to vehicle-treated $3 \times T g-A D^{C D}$ or $3 x T g-A D^{H F D}$ mice, exenatide administration significantly reduced proBDNF signaling in $3 x T g-A D^{C D}$ or $3 x T g-A D^{H F D}$ animals (Fig. $\left.4 a\right)$. In $3 x T g-A D^{H F D}$ mice, the molecule was found to revert the HFD-induced activation of proBDNF/p75NTR signaling (Fig. 4b-d).

\section{Exenatide treatment modulates the inflammatory response in $3 \times T g-A D^{H F D}$ mice}

The HFD is known to promote a pro-inflammatory state through the activation of lipid-mediated signaling ${ }^{41}$. A growing body of evidence indicate that neuroinflammation is present in $A D^{42}$. To address the role of inflammation in our experimental setting, we performed a WB analysis of the pro-inflammatory mediator NF-KB. We also evaluated PPARs, the anti-inflammatory peroxisome proliferator-activated receptor proteins ${ }^{43,44}$. A significant increase in NF-KB levels was found in $3 \times T^{T g-A D}{ }^{H F D}$ mice, a phenomenon reverted by the exenatide administration (Fig. $5 a ; p<0.01$ ). No exenatide-driven effects were observed in $3 x T g-A D^{C D}$ animals (Fig. 5a; $p>0.05$ ). In 3xTg-AD ${ }^{H F D}$ mice, the analysis of the diet-related effects on PPARs revealed a net increase in levels of the PPAR $\alpha$ and PPARy isoforms (Fig. 5b-d; $p<0.001$ ). The effects were inhibited by the exenatide administration (Fig. 5b-d). No effects were observed in exenatide-treated 3xTg$A D^{C D}$ mice (Fig. 5b-d; $p>0.05$ ). In addition, no diet- or drug-related effects were observed when analyzing PPARß/ס levels (Fig. 5c).

\section{Exenatide treatment has no effects on learning and memory performances in $3 x T g-A D^{C D}$ and $3 x T g-A D^{H F D}$} mice

Cognitive effects of HFD and exenatide administration were evaluated in the study groups. To that aim, we employed the MWM test, an experimental setting that evaluates hippocampus-dependent spatial memory ${ }^{45}$. Unexpectedly, compared to $3 x T g-A D^{C D}$ animals, $3 x T g-A D^{H F D}$ mice did not show deficits in learning or long-term memory (Fig. 6a-e; p>0.05). Exenatide treatment resulted in no effects on cognition (Fig. 6a-e; p>0.05). Cognitive performances were also evaluated with the NOR test. The NOR test investigates the hippocampus-dependent spatial memory performances. Compared to the MWM, the NOR is considered more sensitive as the test is less affected by stress-related biases ${ }^{31}$. Compared to $3 \times T^{-}-A D^{C D}$ animals, $3 \times T-A D^{H F D}$ mice did not show differences in the time spent with either the object in the novel location or the DI (Fig. 6f-h; p>0.05). Exenatide administration had no effects on these parameters (Fig. 6f$h ; p>0.05)$. 


\section{Discussion}

In this study, we found that, in 3xTg-AD $\mathrm{HFD}$ mice, 3-month treatment with exenatide enhances BDNF signaling and reduces inflammation. Positive neurotrophic effects were also observed in exenatide-treated $3 \times T g-A D^{C D}$ animals. These findings are in line with previous results supporting a role for GLP-1R agonism in the beneficial modulation of neurotrophic signaling ${ }^{26,28,46}$. Results of the study also revealed distinct molecular effects exerted by HFD on the AD-like background of the 3xTg-AD animals.

\section{Molecular effects of HFD on $3 \times T g-A D$ mice}

HFD administration promoted weight increases and significant IR alterations but did not affect the levels of blood glucose of the mice (Fig. 1). In line with previous findings, compared to $3 \times T g-A D^{C D}$ animals, 3XTg-AD ${ }^{H F D}$ mice developed signs of $I R^{47}$. However, the HFD regimen did not induce a chronic hyperglycemic state or diabetes in the 3xTg-AD animals (Fig. 1h). This result may be explained by the duration of our dietary regimen ${ }^{48}$ as longer ( $>6$ months) HFD is required to produce T2DM.

Previous findings have shown that HFD affects cognition by impairing neurotrophic signaling ${ }^{35}$. In agreement with this notion, in 3xTg-AD mice, HFD promoted a significant decrease in the activation of CREB and BDNF/TrkB signaling (Fig. 3a-c). In our AD model, HFD also decreased levels of pERK5, a BDNFactivated kinase involved in neuronal survival ${ }^{49,50}$, PSD95, a postsynaptic density marker associated with BDNF-driven structural plasticity ${ }^{37}$, and pSyn, a BDNF-activated protein known to promote synaptic vesicle clustering and neurotransmitter release ${ }^{38}$.

In the CNS, the trophic effects of neurotrophins are counteracted by the activity of proneurotrophins, their immature precursor forms. Mature neurotrophins originate from the proteolytic cleavage of pro-neurotrophins, and changes in the balance of mature/immature forms affect neuronal functioning. proBDNF, the precursor form of BDNF, exhibits a high binding affinity for p75NTR ${ }^{51}$. p75NTR,

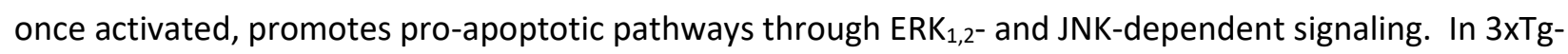
$A D^{H F D}$ mice, we found increased proBDNF levels along with the activation of p75NTR, ERK 1,2 , and JNK (Fig. 4). These results are in line with previous studies showing that HFD and/or IR promote JNK signaling ${ }^{16,32}$.

Our results provide a potential causal link between HFD, the activation of proBDNF signaling, and JNK. The HFD-driven activation of the proBDNF/p75NTR axis was paralleled by a net reduction in levels of pre- and post-synaptic markers like pSyn and PSD95 (Fig. 3e-f). These findings are in agreement with recent evidence indicating that proBDNF-related signaling, occurring via p75NTR, negatively affects neuronal functioning and synaptic remodeling ${ }^{52}$. Our findings also identify, a negative role for $\mathrm{ERK}_{1,2}$. Although ERK $_{1,2}$ activation has been previously considered as a critical step for the BDNF signaling cascade, a growing body of recent evidence has challenged the positive effect of $\mathrm{pERK}_{1,2}$ and shown that the molecules participate in several death-related mechanisms (reviewed in ${ }^{53}$ ). In that regard and in line with our 
previous findings ${ }^{26}$, our results support the idea that ERK $\mathrm{E}_{1,2}$ promotes divergent and bimodal effects and indicate a deleterious HFD-driven activation of $\mathrm{ERK}_{1,2}{ }^{54}$.

Inflammation plays a central role in the pathogenesis of neurodegenerative conditions, including $A D{ }^{42}$. Our findings support the idea that the HFD is a potent trigger of NF-KB, a transcription factor involved in the expression of inflammatory-related cytokines and chemokines (Fig. 5a) ${ }^{55,56}$. In our study, HFD was also found to increase the expression of PPAR $\alpha$ and PPARY (but not PPAR $\beta / \delta$ ), two proteins known to counteract pro-inflammatory pathways (Fig. 5b-d) ${ }^{43}$. This finding may be viewed as a compensatory mechanism set in motion to counteract the HFD-driven inflammatory response ${ }^{44}$. This hypothesis is supported by recent findings showing that, upon active brain inflammation, PPAR $\alpha$ and PPARY but not PPAR $\beta / \delta$ are selectively expressed in microglia ${ }^{57}$ (Fig. $5 b-d$ ).

The HFD did not affect $A \beta$ pathology in our 3xTg-AD mice. This finding diverges from previous reports showing that fat-enriched diets can exacerbate the amyloid pathology ${ }^{33,34}$ but are in line with other studies reporting the lack of effect of HFD on A $\beta$ plaque deposition ${ }^{54,58}$ (Fig. 2a-b). No changes were also observed in p-tau levels when comparing $3 x \operatorname{Tg}-A D^{C D}$ and $3 x \operatorname{Tg}-A D^{H F D}$ mice (Fig. $2 c-d$ ).

\section{Molecular effects of exenatide on $3 \times T g-A D^{C D}$ and $3 \times T g-A D^{H F D}$ mice}

Exenatide was not effective in restoring the insulin sensitivity in the $3 \times T_{g}-A D^{H F D}$ mice, a lack of efficacy likely because the compound promotes regulatory activities only in the presence of patent signs of hyperglycemia or diabetes ${ }^{26,59}$. 3xTg-AD mice at 12 m.o.a. are devoid of metabolic deficits ${ }^{31}$, thereby not offering the pathological background on which exenatide can work.

Previous in vitro and in vivo findings indicate that the GLP1-R agonism exerted by endogenous or exogenous synthetic ligands activates CREB and upregulates BDNF levels ${ }^{60,61}$. We have recently shown that exenatide, administered to adult wild-type mice positively modulates the BDNF-TrkB neurotrophic axis ${ }^{26}$. In this study, exenatide was found to increase the BDNF-related signaling in the hippocampus of $3 \times \mathrm{XT}^{-} \mathrm{AD} \mathrm{D}^{\mathrm{CD}}$ mice and partially restored it to control levels in 3xTg-AD ${ }^{H F D}$ animals (Fig. 3a-c). Given the central role played by BDNF in structural plasticity, it is conceivable that some of the exenatide effects may have worked on synaptic targets. In agreement with this hypothesis, the exenatide-related potentiation of BDNF signaling was paralleled by increases in pERK5, PSD95, and pSyn levels (Fig. 3d-f). The compound promoted only a modest increase in pTrkB levels in $3 \times T^{-}-A D^{H F D}$ mice (Fig. $3 c$ ). Two considerations can explain this result: 1) transgenic AD mice showed impaired TrkB signaling despite the presence of elevated BDNF levels 62. 2) drug-related trophic effects can, at least in part, be mediated by BDNF-independent mechanisms ${ }^{26,63}$. In line with our previous results ${ }^{26}$, exenatide was found to be effective in reducing proBDNF/p75NTR

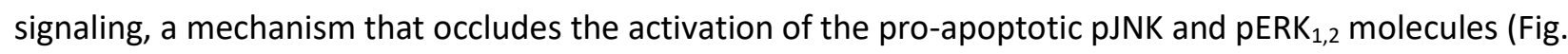
4). 
In $3 \times T g-A D^{H F D}$ mice, exenatide was found to modulate neuroinflammation as shown by the drugdriven reduction of NF-KB levels (Fig. 5a). This finding is supported by several studies showing an antiinflammatory role for GLP-1R agonists in neurodegenerative conditions ${ }^{28,63}$. The drug-mediated reduction of NF-kB was mirrored by a net decrease in PPAR $\alpha$ and PPARY levels (Fig. 5b-d). This result supports the idea that exenatide, by blocking inflammatory signaling, prevents the activation of the endogenous antiinflammatory responses ${ }^{43}$.

In line with our previous findings ${ }^{25}$, exenatide had no effect on $A \beta$ and $p$-tau pathology (Fig. 2a-d).

\section{Effect of exenatide on cognition}

Our findings converge towards potential pro-cognitive effects of exenatide. However, the analysis of the behavioral data failed to reveal significant changes in the memory performances of 3xTg-AD mice. These results are not in line with reports indicating detrimental cognitive effects of HFD in mice ${ }^{34,64 . ~ A ~}$ series of compensatory mechanisms may have produced the lack of cognitive impairment in the study animals. One issue concerns the fact that our 12-month-old (m.o.) 3xTg-AD mice fail to display cognitive deficits. MWM and NOR performances of vehicle-treated 3xTg-AD ${ }^{C D}$ mice did overlap with those observed in age-matched wild-type animals employed by our lab in previous studies [cfr. ${ }^{26,31}$, and data not shown], thereby supporting the notion that our 12-m.o. 3xTg-AD mice were still in a pre-symptomatic phase. Interestingly, this lack of cognitive deficits occurred even in the presence of overt signs of $A \beta$ and $p$-tau pathology (Fig. 2). This result is not surprising as previous findings showed that cognitive performances of the 3xTg-AD mice could be restored or preserved even without decreasing $A \beta$ or $p$-tau levels, thereby indicating the presence of amyloid-independent compensatory mechanisms ${ }^{30,65,66}$. In addition, these mice were able to cope with the HFD-driven metabolic insult. $3 x T g-A D^{H F D}$ mice showed increased peripheral levels of insulin (Fig. 1i) as indicated by the absence of signs of hyperglycemia or diabetes in the study group (Fig. 1c-d and 1h). As insulin actively modulates cognition, it is conceivable that, in the CNS of the 3XTg-AD ${ }^{H F D}$ mice, the enhanced hormone levels may have worked towards the facilitation of a neurotrophic drive that counteracted the impaired BDNF-TrkB axis (Fig. 3a and 3c). This possibility is indirectly indicated by the decreased phosphorylation, and therefore the increased activation, of IRS-1 that we have found in the brain of the $3 x T g-A D^{H F D}$ animals (Fig. 3g), a phenomenon that supports the idea of an ongoing neurotrophic activity exerted by insulin. Lending further support to this hypothesis, the exenatide-driven recovery of the BDNF/TrkB cascade was paralleled by an increase in pIRS-1 levels (Fig. 3), thereby indicating the potential occlusion of any additional insulin-mediated neurotrophic effect.

Exenatide also failed to produce behavioral changes in $3 \times \operatorname{Tg}-A D^{C D}$. The finding was unexpected as the molecule has been described to exert a pro-cognitive activity in WT animals ${ }^{26}$. A possible explanation for this result comes from recent evidence showing that, to promote cognitive improvement in an $A D$ mouse model, BDNF-signaling requires adult neurogenesis ${ }^{67}$. Twelve m.o. 3xTg-AD animals have been 
shown to exhibit signs of impaired hippocampal neurogenesis ${ }^{68,69}$, thereby preventing the beneficial role of exenatide-driven BDNF signaling.

\section{Conclusions}

Despite significant efforts in the field of AD-related therapy, no disease-modifying drugs are currently available. A reconsideration of the "amyloid hypothesis" as well as a more complex view of the disease and the synergistic role played by additional mechanisms is animating the AD community 4,64,70. Growing evidence indicate that the modulation of neurotrophic signaling may be a promising therapeutic avenue to be explored in neurodegenerative conditions and $A D^{71,72}$. Our findings support the need to investigate further exenatide in clinical trials targeting $\mathrm{MCl}$ subjects or patients suffering from the early stage of dementia.

\section{Acknowledgments}

The authors thank all the members of the Molecular Neurology Unit for helpful discussions. The authors are in debt with Paola Siccu and Annalisa Nespoli for technical assistance with IHC.

\section{Funding sources}

SLS is supported by research grants from the Italian Department of Health (RF-2013-02358785 and NET-2011-02346784-1), from the AIRAlzh Onlus (ANCC-COOP), from the Alzheimer's Association - Part the Cloud: Translational Research Funding for Alzheimer's Disease (18PTC-19-602325) and the Alzheimer's Association - GAAIN Exploration to Evaluate Novel Alzheimer's Queries (GEENA-Q-19-596282).

\section{Declaration of interests}

The authors declare no competing interests. The funding sources were not involved in study design, or the collection, analysis, and interpretation of data. The corresponding author had full access to all the data in the study and had final responsibility for the decision to submit for publication.

\section{Author contribution}


bioRxiv preprint doi: https://doi.org/10.1101/487629; this version posted December 4, 2018. The copyright holder for this preprint (which was not certified by peer review) is the author/funder, who has granted bioRxiv a license to display the preprint in perpetuity. It is made available under aCC-BY-NC-ND 4.0 International license.

SLS conceived, designed, and supervised the study. MB performed in vivo treatment, metabolic assays, behavioral testing, and sample collection. VC and AC performed and analyzed WB data. RL performed and analyzed IHC data. MB and AG analyzed and interpreted the data. MB, AG, and SLS wrote the manuscript. All authors approved the final version of the manuscript. 


\section{References}

1. Querfurth HW, LaFerla FM. Alzheimer's disease. N Engl J Med. 2010;362(4):329-344. doi:10.1056/NEJMra0909142.

2. Morris GP, Clark IA, Vissel B. Questions concerning the role of amyloid- $\beta$ in the definition, aetiology and diagnosis of Alzheimer's disease. Acta Neuropathol. 2018;136(5):663-689. doi:10.1007/s00401018-1918-8.

3. Sensi SL. Alzheimer Disease, time to turn the tide. Aging (Albany NY). 2018. doi:10.18632/aging.101581.

4. de la Torre JC. A turning point for Alzheimer's disease? BioFactors. 2012;38(2):78-83. doi:10.1002/biof.200.

5. Nucera A, Hachinski V. Cerebrovascular and Alzheimer disease: fellow travelers or partners in crime? J Neurochem. 2018;144(5):513-516. doi:10.1111/jnc.14283.

6. Smith MA, Rottkamp CA, Nunomura A, Raina AK, Perry G. Oxidative stress in Alzheimer's disease. Biochim Biophys Acta - Mol Basis Dis. 2000;1502(1):139-144. doi:10.1016/S0925-4439(00)00040-5.

7. Boyle PA, Yu L, Wilson RS, Leurgans SE, Schneider JA, Bennett DA. Person-specific contribution of neuropathologies to cognitive loss in old age. Ann Neurol. 2018;83(1):74-83. doi:10.1002/ana.25123.

8. Frölich L, Blum-Degen D, Bernstein H-G, et al. Brain insulin and insulin receptors in aging and sporadic Alzheimer's disease. J Neural Transm. 1998;105(4):423. doi:10.1007/s007020050068.

9. Hoyer S. Causes and consequences of disturbances of cerebral glucose metabolism in sporadic Alzheimer disease: therapeutic implications. Adv Exp Med Biol. 2004;541:135-152. http://www.ncbi.nlm.nih.gov/pubmed/14977212. Accessed May 4, 2017.

10. Craft S. The role of metabolic disorders in Alzheimer disease and vascular dementia: two roads converged. Arch Neurol. 2009;66(3):300-305. doi:10.1001/archneurol.2009.27.

11. Arnold SE, Arvanitakis Z, Macauley-Rambach SL, et al. Brain insulin resistance in type 2 diabetes and Alzheimer disease: concepts and conundrums. Nat Rev Neurol. 2018;14(3):168-181. doi:10.1038/nrneurol.2017.185.

12. Stanley M, Macauley SL, Holtzman DM. Changes in insulin and insulin signaling in Alzheimer's disease: cause or consequence? J Exp Med. 2016;213(8):1375-1385. doi:10.1084/jem.20160493.

13. Cai $\mathrm{W}$, Xue $\mathrm{C}$, Sakaguchi $\mathrm{M}$, et al. Insulin regulates astrocyte gliotransmission and modulates behavior. J Clin Invest. 2018;128(7):2914-2926. doi:10.1172/JCI99366.

14. Craft S. Insulin resistance and Alzheimer's disease pathogenesis: potential mechanisms and implications for treatment. Curr Alzheimer Res. 2007;4(2):147-152. http://www.ncbi.nlm.nih.gov/pubmed/17430239. Accessed February 9, 2015.

15. Craft S, Stennis Watson G. Insulin and neurodegenerative disease: shared and specific mechanisms. 
Lancet Neurol. 2004;3(3):169-178. doi:10.1016/S1474-4422(04)00681-7.

16. De Felice FG, Lourenco M V., Ferreira ST. How does brain insulin resistance develop in Alzheimer's disease? Alzheimer's Dement. 2014;10(1):S26-S32. doi:10.1016/j.jalz.2013.12.004.

17. Campbell JE, Drucker DJ. Pharmacology, Physiology, and Mechanisms of Incretin Hormone Action. Cell Metab. 2013;17(6):819-837. doi:10.1016/j.cmet.2013.04.008.

18. Holst JJ. The Physiology of Glucagon-like Peptide 1. Physiol Rev. 2007;87(4). http://physrev.physiology.org/content/87/4/1409. Accessed May 4, 2017.

19. Abbas T, Faivre E, Hölscher C. Impairment of synaptic plasticity and memory formation in GLP-1 receptor KO mice: Interaction between type 2 diabetes and Alzheimer's disease. Behav Brain Res. 2009;205(1):265-271. doi:10.1016/j.bbr.2009.06.035.

20. Hamilton A, Hölscher C. Receptors for the incretin glucagon-like peptide-1 are expressed on neurons in the central nervous system. Neuroreport. 2009;20(13):1161-1166.

doi:10.1097/WNR.0b013e32832fbf14.

21. During MJ, Cao L, Zuzga DS, et al. Glucagon-like peptide-1 receptor is involved in learning and neuroprotection. Nat Med. 2003;9(9):1173-1179. doi:10.1038/nm919.

22. Hamilton A, Patterson S, Porter D, Gault VA, Holscher C. Novel GLP-1 mimetics developed to treat type 2 diabetes promote progenitor cell proliferation in the brain. J Neurosci Res. 2011;89(4):481489. doi:10.1002/jnr.22565.

23. Porter DW, Irwin N, Flatt PR, Hölscher C, Gault VA. Prolonged GIP receptor activation improves cognitive function, hippocampal synaptic plasticity and glucose homeostasis in high-fat fed mice. Eur J Pharmacol. 2011;650(2-3):688-693. doi:10.1016/j.ejphar.2010.10.059.

24. Perry TA, Greig NH. A new Alzheimer's disease interventive strategy: GLP-1. Curr Drug Targets. 2004;5(6):565-571. http://www.ncbi.nlm.nih.gov/pubmed/15270203. Accessed November 16, 2018.

25. Bomba $M$, Ciavardelli $D$, Silvestri $E$, et al. Exenatide promotes cognitive enhancement and positive brain metabolic changes in PS1-KI mice but has no effects in 3xTg-AD animals. Cell Death Dis. 2013;4(5):e612. doi:10.1038/cddis.2013.139.

26. Bomba M, Granzotto A, Castelli V, et al. Exenatide exerts cognitive effects by modulating the BDNFTrkB neurotrophic axis in adult mice. Neurobiol Aging. 2018;64:33-43. doi:10.1016/j.neurobiolaging.2017.12.009.

27. Hölscher C. New drug treatments show neuroprotective effects in Alzheimer's and Parkinson's diseases. Neural Regen Res. 2014;9(21):1870-1873. doi:10.4103/1673-5374.145342.

28. Athauda D, Foltynie T. The glucagon-like peptide 1 (GLP) receptor as a therapeutic target in Parkinson's disease: mechanisms of action. Drug Discov Today. 2016;21(5):802-818. doi:10.1016/j.drudis.2016.01.013.

29. Athauda D, Foltynie T. Protective effects of the GLP-1 mimetic exendin-4 in Parkinson's disease. 
Neuropharmacology. 2018;136(Pt B):260-270. doi:10.1016/j.neuropharm.2017.09.023.

30. Isopi E, Granzotto A, Corona C, et al. Pyruvate prevents the development of age-dependent cognitive deficits in a mouse model of Alzheimer's disease without reducing amyloid and tau pathology. Neurobiol Dis. 2015;81:214-224. doi:10.1016/j.nbd.2014.11.013.

31. Masciopinto F, Di Pietro N, Corona C, et al. Effects of long-term treatment with pioglitazone on cognition and glucose metabolism of PS1-KI, 3xTg-AD, and wild-type mice. Cell Death Dis. 2012;3(12):e448. doi:10.1038/cddis.2012.189.

32. Kothari $\mathrm{V}$, Luo $\mathrm{Y}$, Tornabene $\mathrm{T}$, et al. High fat diet induces brain insulin resistance and cognitive impairment in mice. Biochim Biophys Acta - Mol Basis Dis. 2017;1863(2):499-508. doi:10.1016/J.BBADIS.2016.10.006.

33. Julien C, Tremblay C, Phivilay A, et al. High-fat diet aggravates amyloid-beta and tau pathologies in the 3xTg-AD mouse model. Neurobiol Aging. 2010;31(9):1516-1531. doi:10.1016/j.neurobiolaging.2008.08.022.

34. Vandal M, White PJ, Tremblay C, et al. Insulin Reverses the High-Fat Diet-Induced Increase in Brain A and Improves Memory in an Animal Model of Alzheimer Disease. Diabetes. 2014;63(12):42914301. doi:10.2337/db14-0375.

35. Molteni R, Barnard RJ, Ying Z, Roberts CK, Gómez-Pinilla F. A high-fat, refined sugar diet reduces hippocampal brain-derived neurotrophic factor, neuronal plasticity, and learning. Neuroscience. 2002;112(4):803-814. http://www.ncbi.nlm.nih.gov/pubmed/12088740. Accessed November 16, 2018.

36. Abdel-Maksoud SM, Hassanein SI, Gohar NA, Attia SMM, Gad MZ. Investigation of brain-derived neurotrophic factor (BDNF) gene expression in hypothalamus of obese rats: Modulation by omega-3 fatty acids. Nutr Neurosci. 2017;20(8):443-448. doi:10.1080/1028415X.2016.1180859.

37. Yoshii A, Constantine-Paton M. BDNF induces transport of PSD-95 to dendrites through PI3K-AKT signaling after NMDA receptor activation. Nat Neurosci. 2007;10(6):702-711. doi:10.1038/nn1903.

38. Greengard P, Valtorta F, Czernik AJ, Benfenati F. Synaptic vesicle phosphoproteins and regulation of synaptic function. Science. 1993;259(5096):780-785.

http://www.ncbi.nlm.nih.gov/pubmed/8430330. Accessed November 19, 2018.

39. Bomfim TR, Forny-Germano L, Sathler LB, et al. An anti-diabetes agent protects the mouse brain from defective insulin signaling caused by Alzheimer's disease- associated A $\beta$ oligomers. J Clin Invest. 2012;122(4):1339-1353. doi:10.1172/JCI57256.

40. Woo NH, Teng HK, Siao C-J, et al. Activation of p75NTR by proBDNF facilitates hippocampal longterm depression. Nat Neurosci. 2005;8(8):1069-1077. doi:10.1038/nn1510.

41. Morselli E, Fuente-Martin E, Finan B, et al. Hypothalamic PGC-1 $\alpha$ Protects Against High-Fat Diet Exposure by Regulating ER $\alpha$. Cell Rep. 2014;9(2):633-645. doi:10.1016/j.celrep.2014.09.025. 
42. Heneka MT, Carson MJ, Khoury J El, et al. Neuroinflammation in Alzheimer's disease. Lancet Neurol. 2015;14(4):388-405. doi:10.1016/S1474-4422(15)70016-5.

43. Daynes RA, Jones DC. Emerging roles of PPARS in inflammation and immunity. Nat Rev Immunol. 2002;2(10):748-759. doi:10.1038/nri912.

44. Benedetti E, Cristiano L, Antonosante A, et al. PPARs in Neurodegenerative and Neuroinflammatory Pathways. Curr Alzheimer Res. 2018;15(4):336-344. doi:10.2174/1567205014666170517150037.

45. Morris RGM, Garrud P, Rawlins JNP, O'Keefe J. Place navigation impaired in rats with hippocampal lesions. Nature. 1982;297(5868):681-683. doi:10.1038/297681a0.

46. Salcedo I, Tweedie D, Li Y, Greig NH. Neuroprotective and neurotrophic actions of glucagon-like peptide-1: an emerging opportunity to treat neurodegenerative and cerebrovascular disorders. $\mathrm{Br} J$ Pharmacol. 2012;166(5):1586-1599. doi:10.1111/j.1476-5381.2012.01971.x.

47. Heydemann A. An Overview of Murine High Fat Diet as a Model for Type 2 Diabetes Mellitus. J Diabetes Res. 2016;2016:1-14. doi:10.1155/2016/2902351.

48. Wang $\mathrm{H}$, Wang $\mathrm{B}$, Yin $\mathrm{H}$, et al. Reduced neurotrophic factor level is the early event before the functional neuronal deficiency in high-fat diet induced obese mice. Metab Brain Dis. 2017;32(1):247257. doi:10.1007/s11011-016-9905-z.

49. Liu L, Cavanaugh JE, Wang Y, Sakagami H, Mao Z, Xia Z. ERK5 activation of MEF2-mediated gene expression plays a critical role in BDNF-promoted survival of developing but not mature cortical neurons. Proc Natl Acad Sci. 2003;100(14):8532-8537. doi:10.1073/pnas.1332804100.

50. Wang Y, Su B, Xia Z. Brain-derived Neurotrophic Factor Activates ERK5 in Cortical Neurons via a Rap1-MEKK2 Signaling Cascade. J Biol Chem. 2006;281(47):35965-35974. doi:10.1074/jbc.M605503200.

51. Lee R, Kermani P, Teng KK, Hempstead BL. Regulation of Cell Survival by Secreted Proneurotrophins. Science (80- ). 2001;294(5548):1945-1948. doi:10.1126/science.1065057.

52. Yang J, Harte-Hargrove LC, Siao C-J, et al. proBDNF negatively regulates neuronal remodeling, synaptic transmission, and synaptic plasticity in hippocampus. Cell Rep. 2014;7(3):796-806. doi:10.1016/j.celrep.2014.03.040.

53. Subramaniam S, Unsicker K. ERK and cell death: ERK1/2 in neuronal death. FEBS J. 2010;277(1):2229. doi:10.1111/j.1742-4658.2009.07367.x.

54. Petrov D, Pedrós I, Artiach G, et al. High-fat diet-induced deregulation of hippocampal insulin signaling and mitochondrial homeostasis deficiences contribute to Alzheimer disease pathology in rodents. Biochim Biophys Acta - Mol Basis Dis. 2015;1852(9):1687-1699. doi:10.1016/J.BBADIS.2015.05.004.

55. Lawrence T. The nuclear factor NF-kappaB pathway in inflammation. Cold Spring Harb Perspect Biol. 2009;1(6):a001651. doi:10.1101/cshperspect.a001651. 
56. Benzler J, Ganjam GK, Pretz D, et al. Central Inhibition of IKK $/$ NF-KB Signaling Attenuates High-Fat Diet-Induced Obesity and Glucose Intolerance. Diabetes. 2015;64(6):2015-2027. doi:10.2337/db140093.

57. Warden A, Truitt J, Merriman M, et al. Localization of PPAR isotypes in the adult mouse and human brain. Sci Rep. 2016;6:27618. doi:10.1038/srep27618.

58. Knight EM, Martins IVA, Gümüsgöz S, Allan SM, Lawrence CB. High-fat diet-induced memory impairment in triple-transgenic Alzheimer's disease (3xTgAD) mice is independent of changes in amyloid and tau pathology. Neurobiol Aging. 2014;35(8):1821-1832. doi:10.1016/J.NEUROBIOLAGING.2014.02.010.

59. Samson SL, Garber A. GLP-1R agonist therapy for diabetes. Curr Opin Endocrinol Diabetes Obes. 2013;20(2):87-97. doi:10.1097/MED.0b013e32835edb32.

60. Ohtake N, Saito M, Eto M, Seki K. Exendin-4 promotes the membrane trafficking of the AMPA receptor GluR1 subunit and ADAM10 in the mouse neocortex. Regul Pept. 2014;190-191:1-11. doi:10.1016/j.regpep.2014.04.003.

61. Gumuslu E, Mutlu O, Celikyurt IK, et al. Exenatide enhances cognitive performance and upregulates neurotrophic factor gene expression levels in diabetic mice. Fundam Clin Pharmacol. 2016;30(4):376-384. doi:10.1111/fcp.12192.

62. Rantamäki T, Kemppainen S, Autio H, et al. The Impact of Bdnf Gene Deficiency to the Memory Impairment and Brain Pathology of APPswe/PS1dE9 Mouse Model of Alzheimer's Disease. KoronyoHamaoui M, ed. PLoS One. 2013;8(7):e68722. doi:10.1371/journal.pone.0068722.

63. Yun SP, Kam T-I, Panicker N, et al. Block of A1 astrocyte conversion by microglia is neuroprotective in models of Parkinson's disease. Nat Med. 2018;24(7):931-938. doi:10.1038/s41591-018-0051-5.

64. White CL, Pistell PJ, Purpera MN, et al. Effects of high fat diet on Morris maze performance, oxidative stress, and inflammation in rats: contributions of maternal diet. Neurobiol Dis. 2009;35(1):3-13. doi:10.1016/j.nbd.2009.04.002.

65. Blurton-Jones $\mathrm{M}$, Kitazawa $\mathrm{M}$, Martinez-Coria $\mathrm{H}$, et al. Neural stem cells improve cognition via BDNF in a transgenic model of Alzheimer disease. Proc Natl Acad Sci. 2009;106(32):13594-13599. doi:10.1073/pnas.0901402106.

66. Knowles JK, Simmons DA, Nguyen T-V V., et al. A small molecule p75NTR ligand prevents cognitive deficits and neurite degeneration in an Alzheimer's mouse model. Neurobiol Aging. 2013;34(8):2052-2063. doi:10.1016/j.neurobiolaging.2013.02.015.

67. Choi SH, Bylykbashi E, Chatila ZK, et al. Combined adult neurogenesis and BDNF mimic exercise effects on cognition in an Alzheimer's mouse model. Science (80- ). 2018;361(6406):eaan8821. doi:10.1126/science.aan8821.

68. Rodríguez JJ, Jones VC, Tabuchi M, et al. Impaired Adult Neurogenesis in the Dentate Gyrus of a 
Triple Transgenic Mouse Model of Alzheimer's Disease. Gwinn K, ed. PLoS One. 2008;3(8):e2935. doi:10.1371/journal.pone.0002935.

69. Rodríguez JJ, Verkhratsky A. Neurogenesis in Alzheimer's disease. J Anat. 2011;219(1):78-89. doi:10.1111/j.1469-7580.2011.01343.x.

70. Herrup K. The case for rejecting the amyloid cascade hypothesis. Nat Neurosci. 2015;18(6):794-799. doi:10.1038/nn.4017.

71. Brem A-K, Sensi SL. Towards Combinatorial Approaches for Preserving Cognitive Fitness in Aging. Trends Neurosci. 2018;41(12):885-897. doi:10.1016/j.tins.2018.09.009.

72. Baazaoui N, Iqbal K. A Novel Therapeutic Approach to Treat Alzheimer's Disease by Neurotrophic Support During the Period of Synaptic Compensation. Perry G, Avila J, Tabaton M, Zhu X, eds. J Alzheimer's Dis. 2018;62(3):1211-1218. doi:10.3233/JAD-170839. 


\section{Figures}

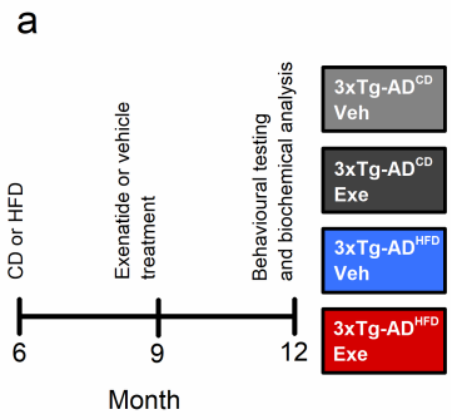

b
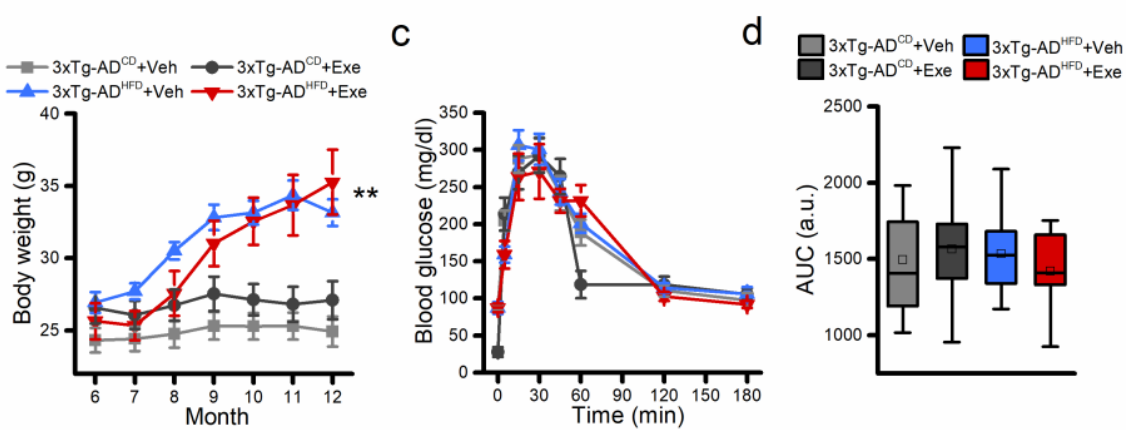

e

f

g
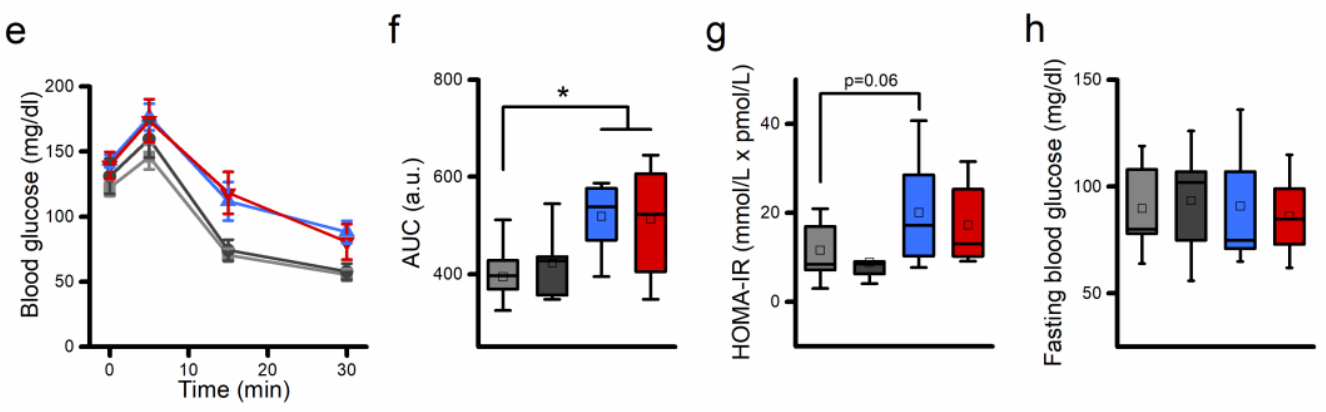

i
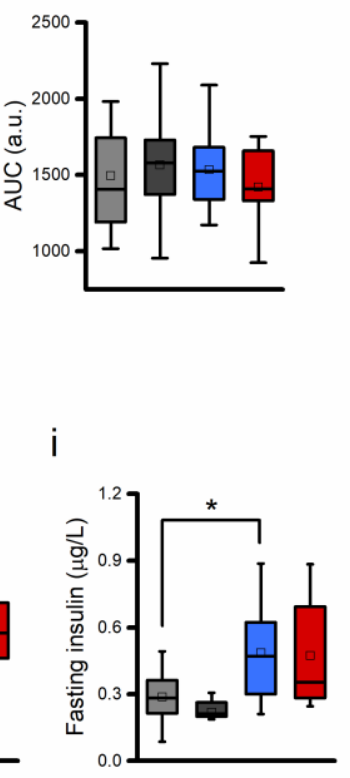

Figure 1. Effects of HFD and exenatide on systemic metabolism. (a) The pictogram illustrates the experimental paradigm employed in the study. (b) Graphs depict body weight changes of 3xTg-AD ${ }^{C D}$ (Veh, $n=13$; Exe, $n=11$ ) or $3 x \operatorname{Tg}-A D^{\text {HFD }}$ (Veh, $n=13$; Exe, $n=9$ ) mice treated with either vehicle or exenatide. (c) Graphs depict the intra-peritoneal GTT (glucose tolerance test) curve of 12 m.o. vehicle- and exenatidetreated $3 x T g-A D^{C D}$ or $3 x T g-A D^{H F D}$ mice. (d) Box charts illustrate the GTT quantifications expressed as area under the curve (AUC). (e) Graphs illustrate the intra-peritoneal ITT (insulin tolerance test) of the four study groups. (f) Box charts illustrate the ITT quantifications expressed as area under the curve (AUC). (g) Box charts indicate changes of the Homeostasis Model Assessment of Insulin Resistance (HOMA-IR) scores in the four groups. (h-i) Box charts illustrate changes in fasting blood glucose and insulin levels. Data are expressed as mean \pm SEM or as box charts where center lines show median, center boxes show mean, box limits indicate $25^{\text {th }}$ and $75^{\text {th }}$ percentiles, and whiskers extend 1.5 times the interquartile range. Means were compared by one-way ANOVA followed by Tukey post-hoc test. * indicates $p<0.05 ;{ }^{* *}$ indicates $p<0.01$. Abbreviations: Veh, vehicle; Exe, exenatide. 
a

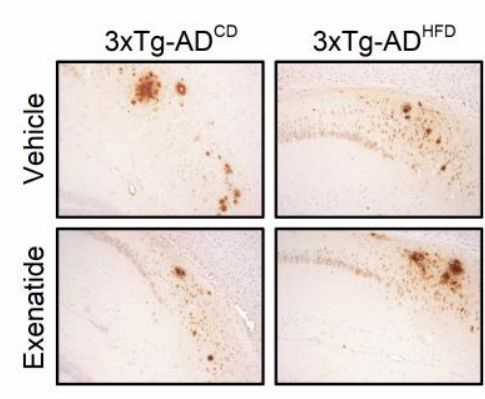

C

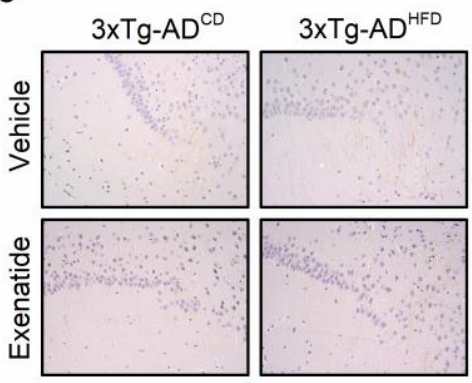

b
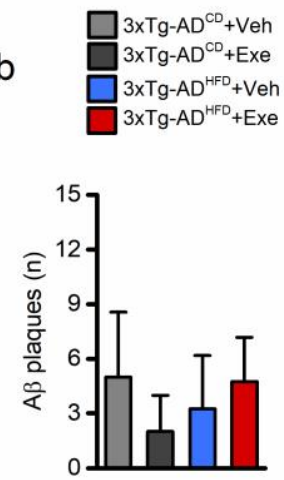

d

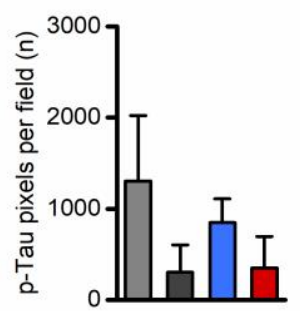

Figure 2. Effects of HFD and exenatide on $A \beta$ and tau pathology. (a) Representative images of $A \beta$ plaque deposition in the hippocampal CA1 region of $3 x \operatorname{Tg}-A D^{C D}$ (Veh, $n=5$; Exe, $n=3$ ) or $3 x \operatorname{Tg}-A D^{H F D}$ (Veh, $n=4$; Exe, $\mathrm{n}=4$ ) mice treated with either vehicle or exenatide. (b) Bar graph depicts $A \beta$ deposit quantification expressed as number of plaques per field. (c) Representative images of intraneuronal p-tau immunoreactivity in the hippocampal CA1 region of $3 x \operatorname{Tg}-A D^{C D}\left(\right.$ Veh, $n=5 ; E x e, n=3$ ) or $3 x \operatorname{Tg}-A D^{H F D}$ (Veh, $n=4$; Exe, $n=3$ ) mice treated with either vehicle or exenatide. Data were analyzed by Kruskal-Wallis test followed by Tamhane post-hoc test. No statistically significant differences were observed among the four study groups. Abbreviations: Veh, vehicle; Exe, exenatide. 

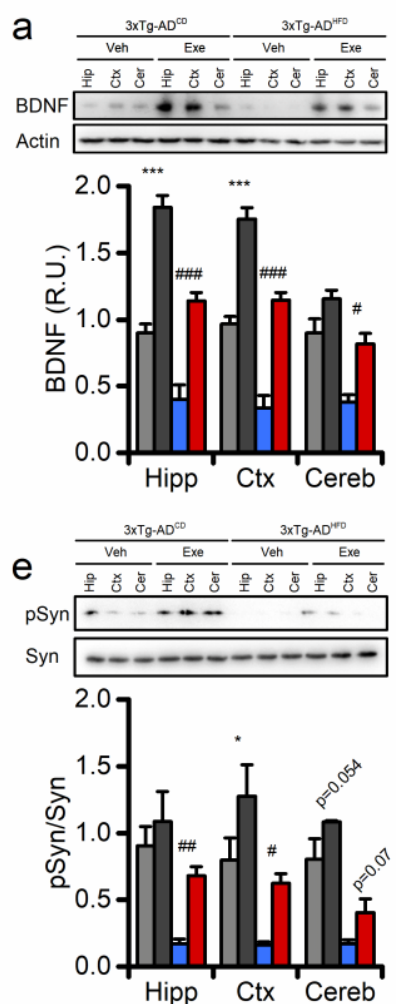

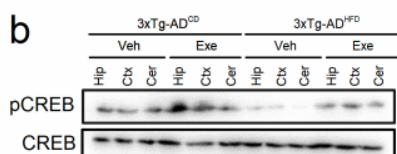

CREB
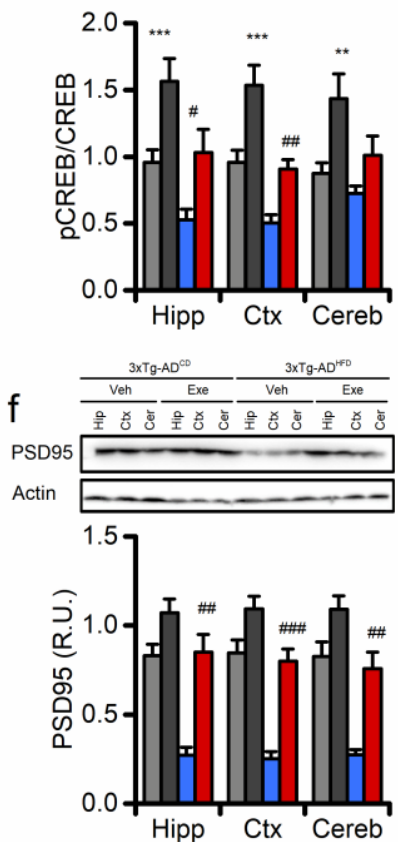
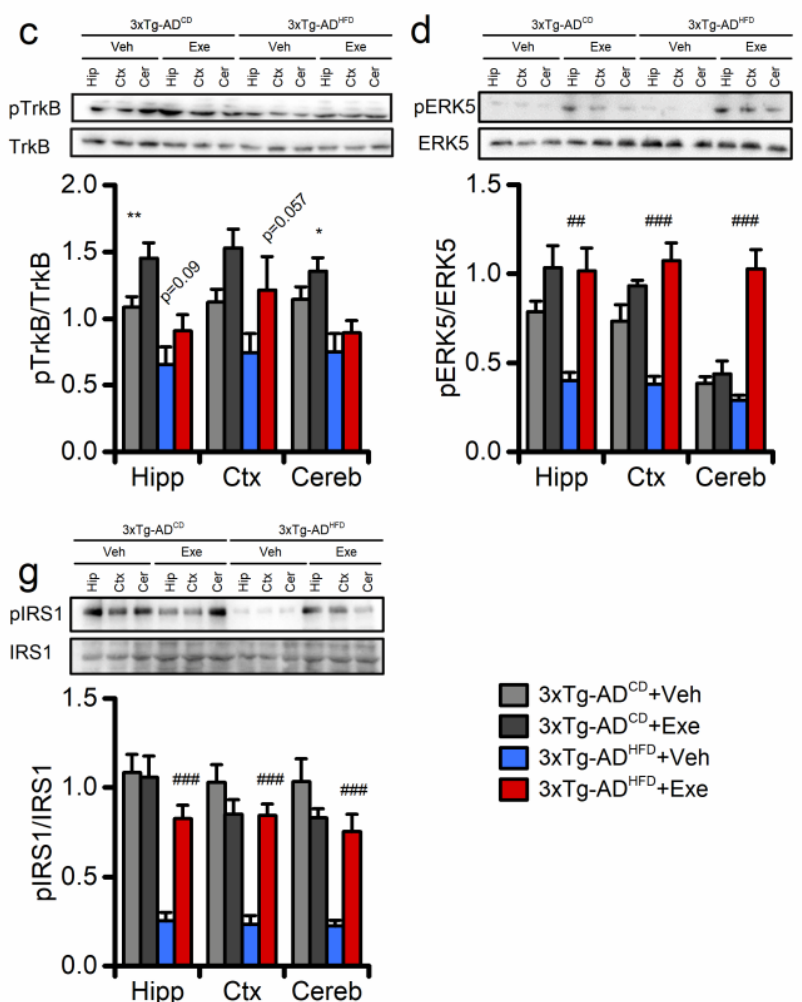

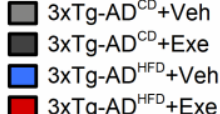

Figure 3. Effects of HFD and exenatide on BDNF neurotrophic signaling. Western blots show exenatideor vehicle-driven effects on BDNF-related signaling in the hippocampus (Hip), cortex (Ctx), and cerebellum (Cer) of 12 m.o. 3xTg-AD ${ }^{C D}$ and 3xTg-AD ${ }^{H F D}$ mice; images are representative of 3-5 independent experiments. (a) Bar graphs depict BDNF levels in the four study groups ( $n=5)$. (b) Bar graphs depict levels of pCREB in the four study groups ( $n=5)$. (c) Bar graphs depict pTrkB levels in the four study groups $(n=5)$. (d) Bar graphs depict pERK5 levels in the four study groups ( $n=5)$. (e) Bar graph depicts levels of pSyn in the four study groups (n=3). (f) Bar graphs depict expression levels of PSD95 in the four study groups ( $n=5)$. Data show mean \pm SEM of relative units (R.U.). Means were compared by one-way ANOVA followed by Tukey post-hoc test. ${ }^{*}$ indicates $p<0.05,{ }^{* *} p<0.01,{ }^{* * *} p<0.001$ of $3 x T g-A D^{C D}+$ Veh versus $3 x T g-A D^{C D}+$ Exe; \# indicates $p<0.05, \# \# p<0.01, \# \# \#<<0.001$ of $3 x \operatorname{Tg}^{-A D^{H F D}}+$ Veh versus $3 \times \operatorname{Tg}^{\mathrm{H}}-\mathrm{AD}{ }^{\mathrm{HFD}}+$ Exe.

Abbreviations: Veh, vehicle; Exe, exenatide. 

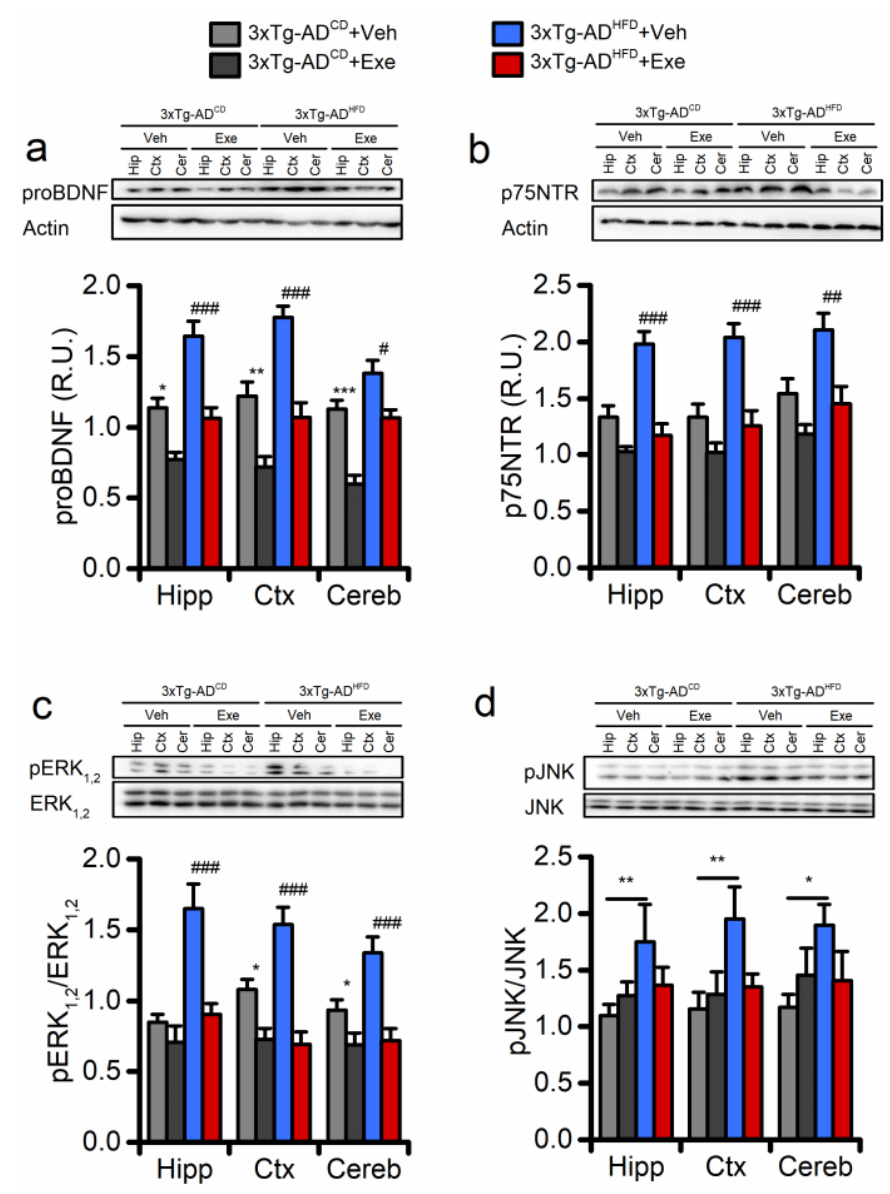

Figure 4. Effects of HFD and exenatide on proBDNF neurotoxic signaling. Western blots show exenatideor vehicle-driven effects on proBDNF-related signaling in the hippocampus (Hip), the cortex (Ctx), and the cerebellum (Cer) of 12 m.o. 3xTg-AD ${ }^{C D}$ and $3 \times T g-A D^{H F D}$ mice; images are representative of 5 independent experiments. (a) Bar graphs depict proBDNF levels in the four study groups $(n=5)$. (b) Bar graphs depict expression levels of p75NTR in the four study groups $(n=5)$. (c) Bar graphs depict $p E R K_{1,2}$ levels in the four study groups $(n=5)$. (d) Bar graphs depict pJNK levels in the four study groups $(n=5)$. Data show mean \pm SEM of relative units (R.U.). Means were compared by one-way ANOVA followed by Tukey post-hoc test. * indicates $p<0.05, * * p<0.01, * * * p<0.001$ of $3 x T g-A D^{C D}+$ Veh versus $3 \times T g-A D^{C D}+E x e ; \#$ indicates $p<0.05, \# \#$ $p<0.01, \# \# \#<<0.001$ of $3 x T g-A D^{H F D}+$ Veh versus $3 x T g-A D^{H F D}+$ Exe. Abbreviations: Veh, vehicle; Exe, exenatide. 

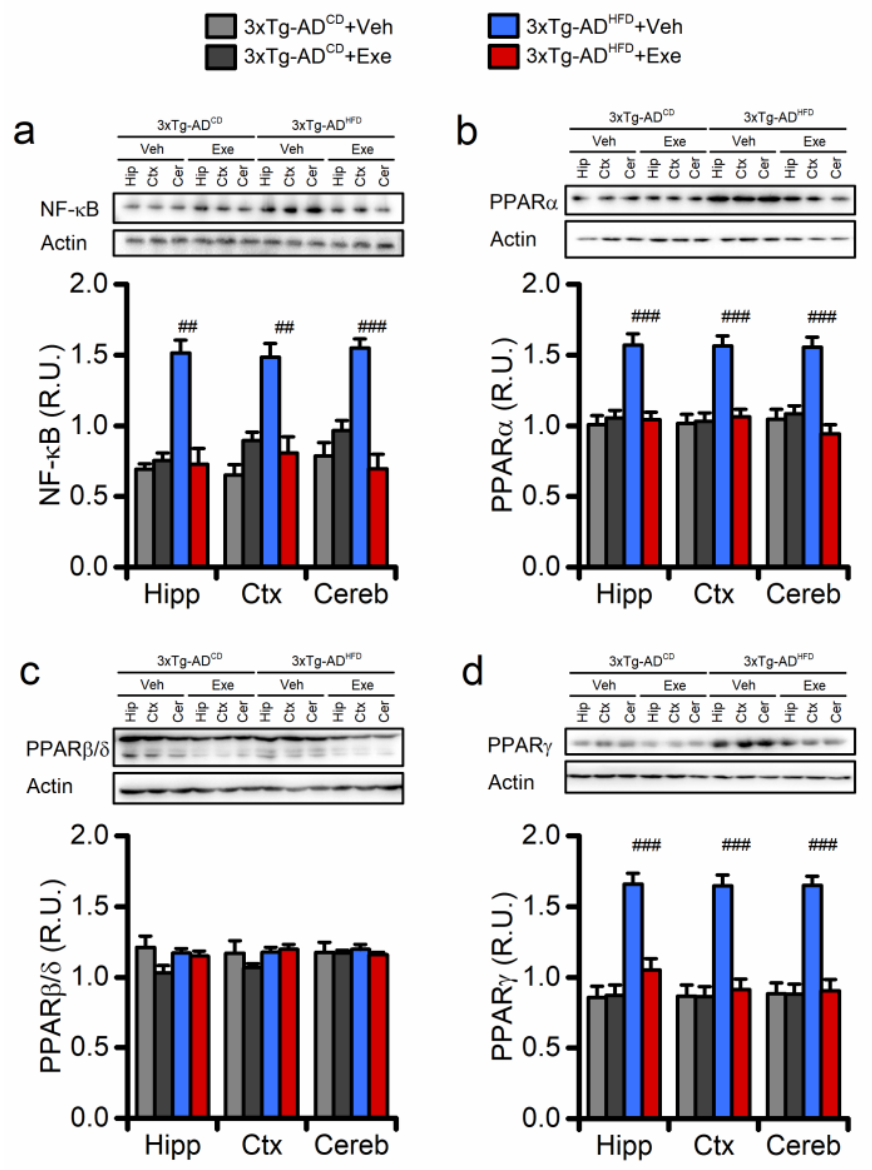

Figure 5. Effects of HFD and exenatide on brain inflammation. Western blots show exenatide- or vehicledriven effects on pro- and anti-inflammatory markers in the hippocampus (Hip), the cortex (Ctx), and the cerebellum (Cer) of 12 m.o. 3xTg-AD ${ }^{C D}$ and 3xTg-AD ${ }^{H F D}$ mice; images are representative of 3-5 independent experiments. (a) Bar graphs depict expression levels of NF-KB in the four study groups ( $n=3)$. (b) Bar graphs depict expression levels of PPAR $\alpha$ in the four study groups $(n=5)$. (c) Bar graphs depict expression levels of PPAR $\beta / \delta$ in the four study groups ( $n=5)$. (d) Bar graphs depict expression levels of PPAR $\gamma$ in the four study groups $(n=5)$. Data show mean \pm SEM of relative units (R.U.). Means were compared by one-way ANOVA followed by Tukey post-hoc test. \#\# indicates $p<0.01$, \#\#\# $p<0.001$ of $3 \times T g-A D^{H F D}+$ Veh versus $3 x T g-A D^{H F D}+$ Exe. Abbreviations: Veh, vehicle; Exe, exenatide. 
a

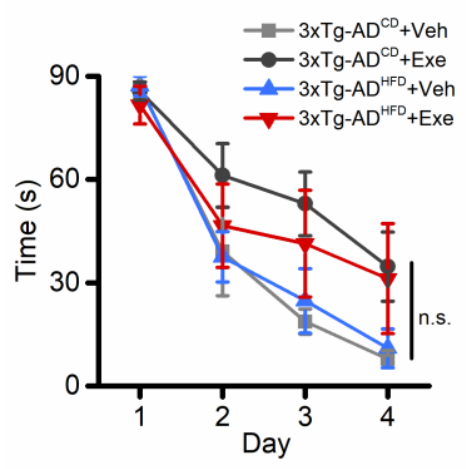

e

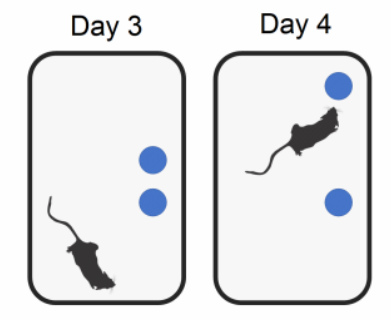

b

f
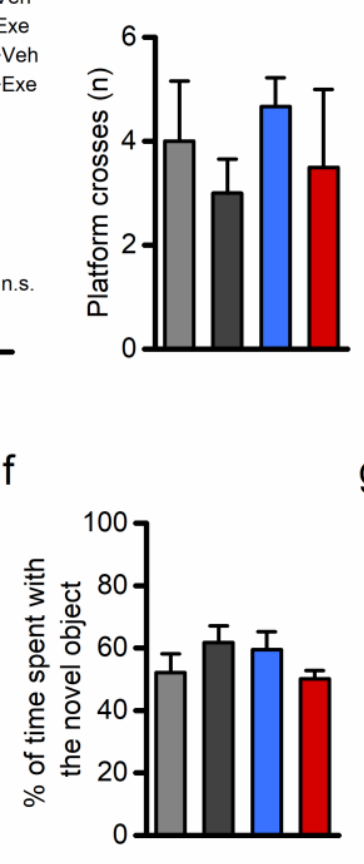

C

g

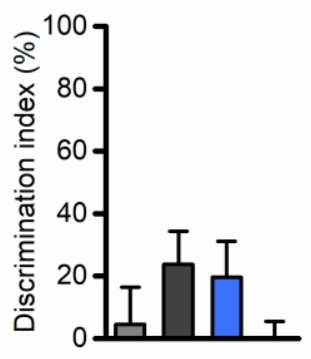

d
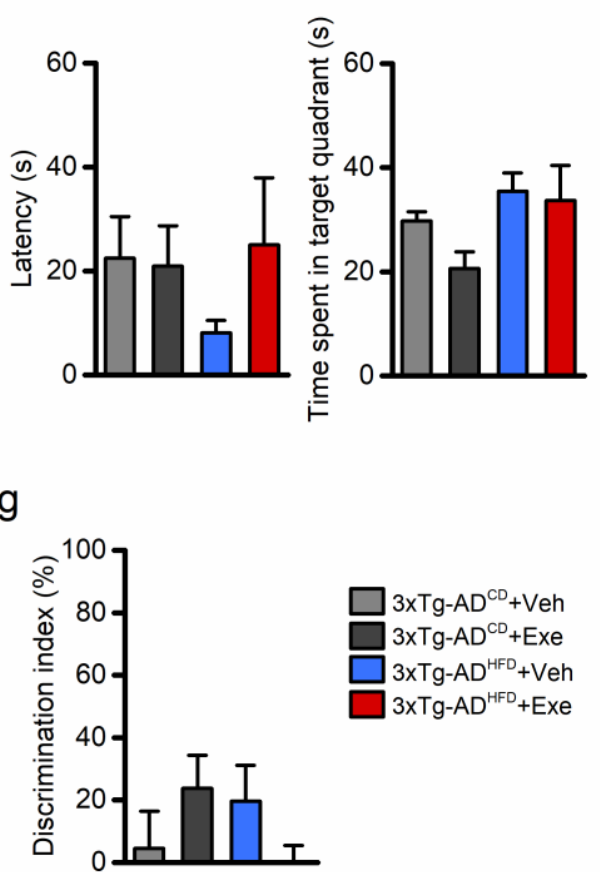

Figure 6. Effects of HFD and exenatide on memory performances. Memory performances were evaluated, in the four study groups, with the Morris Water Maze (MWM) and with the Novel Object Recognition (NOR) tests. (a) The graph depicts the learning curve of $3 x \operatorname{Tg}-A D^{C D}+$ Veh $(n=6), 3 x T g-A D^{C D}+$ Exe $(n=7), 3 x T g-A D^{H F D}+\operatorname{Veh}(n=6)$, and $3 x T g-A D^{H F D}+$ Exe $(n=5)$ mice as assessed upon the 4-day training sessions. Analysis of MWM data revealed no statistically significant differences in learning performances between the four groups $(p>0.05$ ). (b) Bar graphs depict the number of crosses (the number of times each mouse crosses the location where the platform used to be) in the four study groups. (c) Bar graphs depict the latency (the time spent to reach the location where the platform used to be) in the four study groups. (d) Bar graphs depict the time spent in the target quadrant (the quadrant where the platform used to be) in the four study groups. (e) The pictogram illustrates the NOR experimental paradigm employed for testing $3 x T g-A D^{C D}+V e h(n=12), 3 x T g-A D^{C D}+E x e(n=11), 3 x \operatorname{Tg}-A D^{H F D}+V e h(n=12)$, and 3xTg-AD ${ }^{H F D}+E x e(n=9) .(f)$ Bar graphs depict the percentage of time spent with the object moved to a novel location in the four study groups. (g) Bar graphs depict the discrimination index (DI; the difference between time spent exploring novel and familiar objects) in the four study groups. 